\title{
BOAS PRÁTICAS OBSTÉTRICAS INTERMEDIADAS PELO GRUPO GESTAR
}

\section{Leandro da Silva de Medeiros ${ }^{1}$; Andressa Caetano da Veiga ${ }^{2}$; Karla Maria Carneiro Rolim ${ }^{3}$; Francisca Georgina Macedo de Souza ${ }^{4}$; Maria de Lurdes Lomba $^{5}$; Dirce Stein Backes ${ }^{6}$}

\section{RESUMO}

Objetivou-se identificar boas práticas obstétricas, a partir da sistematização de encontros periódicos do grupo GESTAR, que contribuam para a qualificação da rede de atenção integral à saúde materno-infantil. Trata-se de um estudo qualitativo, de caráter exploratório-descritivo, realizado entre outubro/2018 e março/2019, com 15 participantes do grupo GESTAR, por meio de entrevistas individuais, com questões norteadoras. Dos dados analisados resultados três categorias temáticas, quais sejam: Boas práticas obstétricas: compreensões e significados; Boas práticas obstétricas: da teoria à prática profissional; Boas práticas obstétricas: entre o almejado e o proposto. Conclui-se, que embora em passos lentos, as boas práticas obstétricas estão sendo discutidas, assimiladas e implementadas na prática profissional, com vistas à superação do modelo tradicional hegemônico de intervenção obstétrica.

Palavras-chave: Enfermagem obstétrica; Equipe de assistência ao paciente; Humanização de assistência ao parto.

Eixo Temático: Atenção Integral e Promoção à Saúde (AIPS).

\section{INTRODUÇÃO}

Dentre as atuais prioridades do Governo Brasileiro, na área da saúde, estão as políticas públicas voltadas à qualificação da atenção integral à saúde da mulher e da criança, com enfoque na redução da mortalidade materna, neonatal e infantil, na

\footnotetext{
${ }^{1}$ Acadêmico do Curso de Enfermagem da Universidade Franciscana - UFN. Bolsista PPSUS/ FAPERGS. E-mail: leandro.medeiros@ufn.edu.br

2 Fisioterapeuta. Aluna do Mestrado Profissional em Saúde Materno Infantil da Universidade Franciscana - UFN. E-mail: Andressacveiga@gmail.com

${ }^{3}$ Doutora em Enfermagem. Docente do Mestrado Profissional em Tecnologia e Inovação em Enfermagem da Universidade de Fortaleza - UNIFOR. E-mail: karlarolim@unifor.br

${ }^{4}$ Doutora em Enfermagem. Docente do Programa de Pós-Graduação da Universidade Federal do Maranhão - UFMA. E-mail: georginamacedo17@gmail.com

${ }^{5}$ Doutora em Enfermagem. Docente da Escola Superior de Enfermagem de Coimbra/Portugal. E-mail: mlomba@esenfc.pt

${ }^{6}$ Doutora em Enfermagem. Professora do Curso de Enfermagem e do Mestrado Profissional em Saúde Materno Infantil da UFN. Bolsista Produtividade do CNPq. backesdirce@ufn.edu.br
} 
redução dos índices de cesarianas e, sobretudo, no fomento de boas práticas obstétricas (BRASIL, 2017). Tais prioridades visam, sobremaneira, superar o modelo tradicional de intervenção à saúde materno-infantil, o qual tem contribuído para o alcance de resultados maternos e perinatais piores que os encontrados em outros países.

As taxas de cesariana em serviços privados brasileiros atingem indicadores superiores a $80 \%$, o que corresponde ao índice mais alto do planeta. Além das taxas de mortalidade materna que, ainda, são elevadas, a mortalidade neonatal precoce é o componente da mortalidade infantil que vem apresentando a menor queda nos últimos anos e reflete, em parte, a deficiente qualidade da assistência obstétrica (LEAL et al., 2018).

Nessa direção, evidências científicas nacionais demonstram que a medicalização excessiva é, ainda, um dos maiores entraves na rede de saúde materno-infantil. Os partos institucionalizados e realizados por profissionais capacitados chegam a $98 \%$, dos quais cerca de $90 \%$ são realizados por um profissional médico. Persistem intervenções desnecessárias e sem critérios, as quais resultam em taxas de mortalidade materna e infantil elevadas (CARVALHO et al., 2015; SOUZA, 2015).

A Organização Mundial da Saúde (OMS) estimou que, no ano de 2013, aproximadamente $\mathbf{2 8 9} .000$ mulheres, no mundo, perderam a vida durante a gravidez, parto e puerpério. Tais resultados impactaram em uma taxa de mortalidade global de 210 mortes maternas para 100.000 nascidos vivos (WHO, 2016).

Conforme o Relatório dos Objetivos de Desenvolvimento do Milênio, divulgado em 2013, pela Organização das Nações Unidas, o Brasil não atingiu, apesar do declínio na Razão da Mortalidade Materna (RMM), a meta estipulada para o ano de 2015, a qual preconizou reduzir os óbitos maternos para 35 por 100 mil nascidos vivos. Para tanto, foram necessárias ações conjuntas no sentido de estimular mudanças do atual modelo de atenção obstétrica com base na adoção de tecnologias apropriadas para o parto e o nascimento (IPEA, 2014).

Qualificar a atenção obstétrica e reduzir a mortalidade infantil, conforme proposto pela Organização das Nações Unidas, somente será possível mediante o repensar do modelo obstétrico hegemônico e, principalmente, pelo fomento de 
tecnologias apropriadas para o parto e o nascimento. É imprescindível que o profissional da saúde, aqui mais especificamente o Enfermeiro, esteja engajado e comprometido com estas mudanças, no sentido de garantir as boas práticas na atenção ao parto e ao nascimento e, dessa forma, assegurar a qualidade da assistência obstétrica (PEREIRA et al., 2018).

Assim, reconhecendo a necessidade de instaurar um novo pensar e agir profissional, capaz de transcender a pontualidade e a linearidade das práticas hegemônicas e, em consonância com a Agenda de Prioridades de Pesquisa do Ministério da Saúde - APPMS, Eixo 14 - Saúde materno-infantil (BRASIL, 2018), o Grupo GESTAR, tem por finalidade fomentar discussões teórico-práticas que contribuam para a qualificação da rede de atenção à saúde materno-infantil. Com base no exposto, objetivou-se identificar boas práticas obstétricas, a partir da sistematização de encontros periódicos do grupo GESTAR, que contribuam para a qualificação da rede de atenção integral à saúde materno-infantil.

\section{METODOLOGIA}

Trata-se de uma pesquisa qualitativa, de caráter exploratório-descritivo desenvolvida com os integrantes do grupo de gestantes - GESTAR, a partir da sistematização de encontros periódicos quinzenais de 02 horas, com vistas à ampliação das boas práticas obstétricas. Defende-se, que as boas práticas de gestão e de qualificação da atenção integral à saúde materno-infantil se ampliam, na medida em que os atores sociais se encontram para dialogar acerca das concepções vigentes e, a partir de então, construir novos pactos de convivência e transformação dos cenários.

A pesquisa qualitativa possibilitará não apenas encontrar novos achados de pesquisa, senão encontrar estratégias coletivas para o desenvolvimento de saberes, tecnologias e boas práticas em saúde que contribuam para qualificação da rede de atenção integral à saúde materno-infantil.

Os dados foram coletados entre outubro/2018 e março/2019, por meio de entrevistas individuais com integrantes do grupo GESTAR, após a sistematização dos encontros, nos quais foram abordados os seguintes temas: Lei do acopanhante; $O$ que são boas práticas obstétricas; Pré-natal odontológico; Pré-natal psicólogico; 0 
Enfermeiro no trabalho de parto, parto e nascimento; Aromaterapia no processo de nascimento; Direitos de gestantes e puérperas; O períneo e o parto. Foram incluídos no estudo todos os integrantes do grupo, maiores de 18 anos, que aceitaram participar do estudo, após a assinatura do Termo de Consentimento Livre e Esclarecido.

O corpus do estudo foi composto por 15 participantes, dos quais: sete residentes de Enfermagem Obstétrica, cinco estudantes dos Cursos da área da saúde e três gestantes. As entrevistas foram conduzidas por meio de questões norteadoras: o que você entende por boa prática obstétrica? Como e quando você identifica as boas práticas obstétricas em seu fazer diário? Quais boas práticas podem/devem ser desenvolvidas para qualificar a rede de atenção integral à saúde materno-infantil?

Os dados de pesquisa foram analisados com base na técnica de análise de conteúdo temática preconizada por Bardin. A mesma consiste em descobrir os núcleos de sentido que compõem uma comunicação, cuja presença ou frequência acrescentem perspectivas significativas ao objeto de estudo em questão. A noção da temática está associada a uma afirmação que diz respeito a um determinado assunto, podendo ser apresentada por uma palavra, frase ou ideia.

Desse modo, a operacionalização do processo de análise seguirá as três etapas do método. Na primeira etapa, denominada de pré-análise, se busca fazer uma leitura exaustiva dos dados, seguida da organização do material e a formulação de hipóteses. Na sequência, será realizada a exploração do material, ou seja, se busca codificar os dados brutos. Na terceira e última fase, os dados serão interpretados e delimitados em eixos temáticos pela compreensão dos significados estabelecidos (BARDIN, 2011).

Ressalta-se, que o estudo faz parte de uma pesquisa maior intitulado "Qualificação da rede de atenção à saúde materno-infantil na região central do Rio Grande do Sul" que tem parecer favorável do Comitê de Ética em Pesquisa (CEP) sob o número de protocolo: 4.253.922.

\section{RESULTADOS E DISCUSSÕES}

Dos dados organizados e analisados resultados três categorias temáticas, quais sejam: Boas práticas obstétricas: compreensões e significados; Boas práticas 
obstétricas: da teoria à prática profissional; Boas práticas obstétricas: entre o almejado e o proposto.

\subsection{Boas práticas obstétricas: compreensões e significados}

Ao conceituar a humanização do parto, pode-se entendê-la como um movimento pautado na singularidade e autonomia feminina, valorizando o protagonismo da mulher e permitindo maior congruência do cuidado com o sistema cultural de crenças e valores.

Nesta sequência alguns participantes, em especial os residentes de Enfermagem Obstétrica, exprimiram a relação direta do protagonismo da gestante/família com as boas práticas, no que se refere à autonomia na escolha do tipo de parto, a posição, o local de nascimento e sobretudo na singularização de cada evento da mulher, de acordo com as falas a seguir:

Acredito que tais relação ao respeito e direito das gestantes, desde do prénatal até seu nascimento. Ter boas práticas deve ser poder ofertar um bom atendimento e atenção às suas necessidades (P5).

Boas práticas obstétricas é quando a mulher/parturiente tem livre escolha em relação ao seu parto, em como a mulher deseja ganhar, o local, a posição e o profissional respeita, orienta e apoia as escolhas que essa mulher faz (P6).

Trata-se de uma prática em que a mulher e família são protagonistas do processo de parturição. Boa prática obstétrica remete a um "evento natural" e não patológico. A boa prática obstétrica remete, ainda, à atenção integral, no qual todos são atores e protagonistas (P10).

Práticas que respeitem o desejo da mulher que está sendo atendida, sem como que respeitem o processo fisiológico de trabalho de parto e parto (P13).

Evidenciou-se, na fala dos participantes, que as boas práticas obstétricas possuem significados que englobam desde o cuidado direto à mãe e o bebê, isto é, gestação ao puerpério, às condições do ambiente e do processo de trabalho.

Para os estudantes, as boas práticas estão fortemente associadas ao cuidado integral, capaz de possibilitar vivências positivas e afetivas à mãe e ao bebê, já sendo um momento proveitoso para que a equipe de saúde possa demonstrar atenção, interesse e disponibilidade, pretendendo conhecer e compreender as expectativas da parturiente e sua família, aclarando suas dúvidas relacionadas à gestação e ao parto. 
Associam-se, ainda, a um ambiente favorável e estimulador, capaz de singularizar o cuidado, com base nas necessidades e expectativas, o que deve ser realizado no sentido de melhorar a assistência prestada e torná-la humanizada.

Boas práticas são práticas assistênciais consideradas adequadas e que proporcionam vivências positivas e efetivas na realização do cuidado (P3).

Poder dar a mãe e ao bebê o melhor cuidado e amparo nas dificuldades enfrentadas. Como também explicar as mães o que elas precisam saber, como agir, oferecer as melhores opções para um parto e uma gestação saudável (P8).

Boas práticas se referem em ações ofertadas que estejam pautadas no cuidado integral, no respeito ao sujeito e ao seu direito de escolha, sem desconsiderar os fatores subjetivos (P11).

As boas práticas obstétricas foram associadas, também, às evidências científicas, ou seja, às experiências já comprovadas no campo da ciência e que comprovam a sua eficácia. Um participante se referiu, ainda, às melhores evidências disponíveis, sobretudo, às defendidas pela Organização Mundial da Saúde.

Práticas que sejam benéficas tanto para mãe quanto para o bebê, de acordo com as vontades da mãe, baseando-se em evidências científicas (P1).

Práticas aplicadas no pré-natal, parto e pós-parto que estão embasadas em estudos que comprovem eficácia, principalmente às apoiados pela OMS (P2).

É a prática que respeita a autonomia da mulher e da família no processo de parturição com o atendimento baseado nas melhores evidências cientificas disponíveis (P4).

\subsection{Boas práticas obstétricas: da teoria à prática profissional}

Em relação ao seu fazer diário, os participantes conseguem identificar boas práticas obstétricas que vão desde as atitudes e posturas profissionais, a educação em saúde e a assistência acolhedora e atenciosa na hora do parto, dentre outras técnicas não farmacológicas para o alívio da dor, como a deambulação e o posicionamento livre, na visão dos entrevistados, são práticas que devem ser realizadas no sentido de melhorar a assistência prestada.

Devido as orientações, auxilio para beneficiar o trabalho de parto, informações passadas a gestante e seu acompanhante, e esclarecimento de dúvidas (P1).

Dando opção de escolha e comunicação direta com a família. Realizando campleamento oportuno de cordão, contato pele a pele, amamentação na 
primeira hora, comunicando cada ação respeitando opções do paciente. Avaliação detalhada de cada caso individualmente (P2).

Quando eu garanto a autonomia da mulher, quando oferto tecnologias de alivio da dor, proporciono a liberdade de posição, oferta de alimentos e líquidos, garantia do acompanhante (P3).

As boas práticas são identificadas no meu fazer diário quando há algum residente plantonista que luta para realização das mesmas (P13).

Compreende-se, a partir das falas, que a utilização de técnicas de conforto da dor e a participação ativa da mulher são práticas vinculadas à humanização do parto. Tais práticas tendem, também, a contribuir para que a parturiente tenha mais liberdade e autonomia.

Quando a mulher tem livre escolha sobre seus desejos, na hora do parto, na orientação durante consultas do pré-natal quando há pela parte dos profissionais respeito e orientação sobre a importância do parto normal e quando as dúvidas dessas mulheres são esclarecidas (P6).

Principalmente quando pacientes relatam que tiveram as suas escolhas respeitadas; em uma assistência ofertada a partir de ações baseadas em evidências, fatos que implica a redução de violências (P11).

Alguns participantes fizeram referência, também, às boas práticas que evidenciam no âmbito da atenção primária em saúde, mais especificamente às relacionadas ao pré-natal. Ressaltam, nessa direção, a importância das informações e encaminhamentos corretos, o empoderamento da gestante para a escolha autônoma do tipo de parto, dentre outros tópicos.

No pré-natal adequado, na identificação de riscos gestacionais e encaminhamentos em tempo oportuno, no atendimento respeitoso a mulher e a família. $\mathrm{Na}$ aplicação do conhecimento cientifico no atendimento ao trabalho de parto (P4).

No atendimento pré-natal, na escuta a gestante, na oferta de informação, na humanização às gestantes (P5).

As mulheres necessitam receber às informações em relação aos procedimentos a que forem submetidas ou de quaisquer decisões que forem tomadas. Tal atitude auxilia as parturientes a conhecerem e saberem o que será realizado com elas, gerando menos medos e receios, auxiliando o trabalho de parto e parto.

No pré-natal quando garanto a informação a gestante; preenchimento adequado da caderneta; Trabalho de parto - respeito a mulher, garantia de 
segurança; prática baseada em evidência; Puerpério - Garantia da possibilidade de voz da puérpera, apoio e auxílio neste momento (P15).

Outros participantes, ainda, fizeram menção a atuação profissional, ou seja, as boas práticas relacionadas ao empoderamento do profissional. Compreendem, nessa direção, que para emancipar a gestante o profissional precisa estar empoderado com conhecimento técnico-científico.

Quando o profissional tem o domínio do assunto e procura sempre saber mais ele consequentemente terá boas práticas nesse campo (P7).

Eu identifico as boas práticas sempre que vejo um enfermeiro apropriado do conhecimento obstétrico; uma mulher emancipada e protagonista do processo de parturição; um aluno desejoso de mudanças; profissionais abertos e flexíveis para um novo agir e pensar (P10).

O uso rotineiro de práticas já consideradas obsoletas, de acordo com os participantes, deve ser evitado, apenas utilizando-se de práticas validadas pelas melhores evidências científicas, aliando a arte com a ciência, visando um nascimento seguro para mãe e bebê, com o mínimo de intervenções desnecessárias.

\subsection{Boas práticas obstétricas: entre o almejado e o proposto}

Dentre as boas práticas podem e/ou devem ser desenvolvidas para qualificar a rede de atenção integral à saúde materno-infantil se destacam os cuidados com o recém-nascido, qualificado pré-natal, ambientes agregadores e estimuladores, sensibilização da equipe, competência técnico-científica dos profissionais e, sobretudo, um novo modelo de gestão da rede, com novos referenciais teóricopráticos, na perspectiva da multiprofissionalidade, conforme segue:

Alcançar uma rede linear entre profissionais diante das práticas realizadas em obstetrícia, cuidados com recém-nascido para qualificar e humanizar a assistência (P1).

Empoderamento das mulheres em geral na sociedade. Bom pré-natal e avaliação minuciosa. Ambiente tranquilizante no parto, avaliação adequada no trabalho de parto, parto assistido conforme evidências cientificas. Sensibilização da equipe e frequente atualização (P2).

Dentre as boas práticas obstétricas destaco: Novo modelo de gestão da rede de atenção integral; Novas referências de pensamentos, na perspectiva multiprofissional; Maior integração/interação, diálogo entre os serviços que compõe a rede; Grupos de gestantes com metodologias horinzontalizadas que instigam a mulher/família para serem protagonistas do parto; Atualização profissional, na perspectiva interdisciplinar (P10). 
Atendimento multiprofissional; acessibilidade aos serviços de saúde e profissionais as gestantes e família; Conhecimento/acesso a informação de qualidade; qualidade dos profissionais (P14).

Alguns participantes fizeram referência às tecnologias não farmacológicas de alívio da dor fundamentadas em evidências científicas, além do acesso aos serviços de saúde e o atendimento singularizado às necessidades de cada usuária e família.

$\mathrm{Na}$ assistência obstétrica são exemplos de boas práticas liberdade de posição, tecnologias não farmacológicas de alívio de dor, apoio emocional, ingesta de alimentos e líquidos e clampeamento oportuno, contato pele a pele, amamentação na primeira hora de vida, dentre outros (P3).

Qualificação do pré-natal; acesso aos serviços e referenciação a maternidade; engajamento de todas as ênfases profissionais nas práticas baseadas em evidencias; melhoria dos serviços com profissionais capacitados melhoria da ambiência (P4).

Melhorar o atendimento, respeitar suas singularidades, ofertar suas informações, respeitar seus direitos e desejos nesse período de gestação (P5).

Outros participantes, ainda, fizeram referência à participação do acompanhante no processo de gestação e parturição, além da atuação multiprofissional em casas de parto, qualificação dos profissionais com base em propostas horizontalizadas e humanizadas de educação permanente em saúde, dentre outros aspectos relacionados ao financiamento.

Direito ao acompanhante presente junto com a parturiente, direito a ter suas dúvidas esclarecidas de maneira respeitosa sobre parto normal e cesárea para essa mulher ter direito à livre escolha em relação ao seu parto (P6).

Respeito a parturiente e sua rede de apoio afetivo-família; Respeito a hora de ouro; Repasse de informações com relação aos seus direitos; Manejo adequado da amamentação entre outros (P11).

Multidisciplinaridade desde o pré-natal até o pós-parto; Acompanhamento qualificado; profissionais qualificados, atualizados; práticas baseadas em evidência científica das melhores condutas; Respeito a fisiologia (P13).

Qualificação dos profissionais quanto a planejamento reprodutivo; qualificação pré-concepcional, pré-natal, puerpério e infância; Investimento nos serviços de saúde (financeiramente); Educação permanente; Elaboração de fluxograma municipal e protocolos clínicos e assistências (P15). 


\section{DISCUSSÃO}

Evidências científicas apontam que o emprego das boas práticas de atenção ao parto e nascimento, embora incipientes, podem ser consideradas essenciais para o desenvolvimento de nova cultura na assistência obstétrica. As boas práticas, dentre outros benefícios, desvinculam o modelo tecnocrático de atendimento ao binômio mãe-bebê e estimulam as práticas baseadas em evidências científicas (PEREIRA et al., 2018).

As boas práticas baseadas em evidências científicas possibilitam, nesse percurso, a reflexão crítica, com foco na singularidade e na multidimensionalidade humana. Buscam transcender a linearidade das ações profissionais ao proporcionar um ambiente acolhedor, no qual todos são atores e propulsores de novas formas de ser e agir (CÔRTES et al., 2015). Reconhece-se, que o modelo tradicional de intervenção à saúde materno infantil, no país, tem contribuído para os resultados maternos e perinatais piores que os encontrados em outros países com índices de desenvolvimento socioeconômico igual ou menor.

Como exemplo, pode-se citar as taxas de cesariana nos serviços privados, os quais atingem indicadores superiores a $80 \%$, o que corresponde ao índice mais alto do planeta. Além disso, as taxas de mortalidade materna ainda são elevadas. Para transformar esse cenário, vários setores sociais têm se articulado com o objetivo de implantar um novo modelo de assistência ao parto e nascimento, tendo como um dos objetivos devolver o protagonismo da mulher no momento do parto e nascimento (PICHETH; CRUBELLATE; VERDU, 2018)

A humanização do parto, reconhecida como uma política pública de saúde, foi compreendida pelos participantes entrevistados como um conjunto de condutas, atitudes e posturas, ausentes de julgamentos e baseadas no diálogo, na empatia e no acolhimento da usuária e de seus familiares, o fornecimento de orientações e informações quanto às condutas a serem adotadas, a valorização da parturiente e a sua personificação enquanto sujeito de direitos e necessidades (POSSATI et al., 2017).

Para eles, a humanização do parto envolve, ainda, a realização de procedimentos comprovados cientificamente benéficos à saúde materno-infantil e a 
constante atualização profissional neste meio. Nesse processo, a humanização implica no respeito às escolhas, individualidades e singularidades de cada parturiente. Percebe-se, portanto, que as enfermeiras têm conhecimento sobre as políticas e recomendações do Ministério da Saúde e da OMS e, ainda, acreditam que a humanização se encontra em um processo lento, permeada por muitos desafios (CARVALHO et al., 2015).

\section{CONCLUSÃO}

Conclui-se, que embora em passos lentos, as boas práticas obstétricas estão sendo discutidas, assimiladas e implementadas na prática profissional, com vistas à superação do modelo tradicional hegemônico de intervenção obstétrica.

Espera-se, que os resultados deste estudo possam possibilitar reflexões sobre a importância de valorizar a singularidade e o protagonismo da mulher no processo gestacional. Espera-se, também, que outros grupos de discussão possam ser fomentados, no sentido de ampliar as discussões sobre as boas práticas obstétricas e delinear estratégias teórico-práticas condizentes com as diversas realidades.

As limitações deste estudo estão associadas à baixa adesão e participação de gestantes nesta pesquisa. Sugere-se, nessa direção, a realização de encontros nos serviços de saúde, por ocasião das consultas gestacionais.

\section{REFERÊNCIAS}

BARDIN, L. Análise de conteúdo. Lisboa: Edições 70, 2011.

BRASIL. Departamento de Informática do Sistema Único de Saúde - Datasus. Nascidos vivos Brasil. [Internet]. 2017. Disponível em: http://tabnet.datasus.gov.br/cgi/tabcgi.exe?sinasc/cnv/nvuf.def Acesso em: 10 de Jun. 2019.

BRASIL. Ministério da Saúde. Secretaria de Ciência, Tecnologia e Insumos Estratégicos. Departamento de Ciência e Tecnologia. Agenda de Prioridades de Pesquisa do Ministério da Saúde - APPMS. Brasília, 2018.

CÔRTES, C. T. et al. Implementation methodology of practices based on scientific evidence for assistance in natural delivery: a pilot study. Revista da Escola de Enfermagem da USP, v. 49, n. 05, 2015. https://doi.org/10.1590/S0080$\underline{623420150000500002 .}$ 
CARVALHO, E. M. P.; GOTTEMS, L. B. D.; PIRES, M. R. G. M. Adesão às boas práticas na atenção ao parto normal: construção e validação de instrumento. Rev. esc. enferm. USP, v. 49, n. 6, p. 889-897, 2015. http://dx.doi.org/10.1590/S0080$\underline{623420150000600003 .}$.

INSTITUTO DE PESQUISA ECONÔMICA APLICADA - IPEA. Secretaria de planejamento e investimentos estratégicos; Grupo técnico para o acompanhamento dos ODS. Objetivos de Desenvolvimento do Milênio: Relatório Nacional de Acompanhamento. Brasília: Ipea: MP, SPI, 2014.

LEAL, M. C. et al. Saúde reprodutiva, materna, neonatal e infantil nos 30 anos do Sistema Único de Saúde (SUS). Ciência \& Saúde Coletiva, v. 23, n. 6, 2018. https://doi.org/10.1590/1413-81232018236.03942018.

POSSATI, A. B. et al. Humanization of childbirth: meanings and perceptions of nurses. Escola Anna Nery, v. 21, n. 4, 2017. https://doi.org/10.1590/2177-9465-EAN-2016$\underline{0366 .}$.

PEREIRA, S. B. et al. Good practices of labor and birth care from the perspective of health professionals. Rev Bras Enferm, v. 71 (Suppl 3):1313-9, 2018. DOI: http://dx.doi.org/10.1590/0034-7167-2016-0661.

PICHETH, S. F.; CRUBELLATE, J. M.; VERDU, F. C. A transnacionalização do parto normal no Brasil: um estudo das últimas cinco décadas.

História, Ciências, Saúde-Manguinhos, v. 25, n. 4, pág. 1063-1082, 2018. https://doi.org/10.1590/S0104-59702018000500009.

SOUZA, J. P. A mortalidade materna e os novos objetivos de desenvolvimento sustentável (2016-2030). Rev Bras Ginecol Obstet. V.37, n.12, p.549-51, 2015.

WORLD HEALTH ORGANIZATION. WHO. Maternal mortality [Internet]. 2016. Disponível em: http://www.who.int/mediacentre/factsheets/fs348/en/ Acesso em: 15 Jun. 2019. 